\title{
Perfil laboral, comportamentos e saúde segundo análise de gênero entre professores da rede pública
}

\author{
Work profile, behaviors and health according to gender analysis among public school \\ teachers
}

Tatiana Almeida de Magalhães ${ }^{1}$

Marise Fagundes Silveira ${ }^{2}$

Jairo Evangelista Nascimento ${ }^{3}$

Marta Raquel Mendes Vieira ${ }^{4}$

Emerson Willian Santos de Almeida ${ }^{5}$

Andréa Maria Eleutério de Barros Lima Martins ${ }^{6}$

Efigênia Ferreira e Ferreira ${ }^{7}$

Desirée Sant ${ }^{\prime}$ Ana Haikal ${ }^{8}$

Resumo: Objetivou-se identificar possíveis diferenciais de gênero entre professores segundo o perfil laboral, comportamentos e saúde. Trata-se de um estudo de prevalência usando amostra probabilística por conglomerados de docentes do ensino básico da rede pública de um município norte mineiro. Foram estimadas prevalências e análises bivariadas corrigidas pelo desenho amostral. Dos 745 docentes investigados, as mulheres representam ampla maioria

\footnotetext{
${ }^{1}$ Mestre em Ciências da Saúde (Unimontes), Doutoranda (PPGCS/Unimontes), Minas Gerais. Brasil. tatimagmoc@gmail.com (D) https://orcid.org/0000-0001-8371-863X.

${ }^{2}$ Doutora em Saúde Coletiva (UNIFESP), Professora (PPGCS/Unimontes), Minas Gerais. Brasil.

$\triangle \underline{\text { ciaestatistica@yahoo.com.br (i) http://orcid.org/0000-0002-8821-3160. }}$

${ }^{3}$ Doutor em Ciências da Saúde (Unimontes), Professor (Faculdades Unidas do Norte de Minas /FUNORTE), Minas Gerais. Brasil. $₫$ jairomenmoc@gmail.com. (D) https://orcid.org/0000-0003-4010-3971.

${ }^{4}$ Doutora em Ciências da Saúde (Unimontes), Enfermeira (Superintendência Regional de Saúde de Montes Claros), Minas Gerais. Brasil. $₫$ martaraquelmendes@hotmail.com. 1 https://orcid.org/0000-0001-5185-5381.

${ }^{5}$ Graduação em Enfermagem (Unimontes), Mestrando (Programa de Pós-Graduação em Enfermagem Fundamental- Escola de Enfermagem de Ribeirão Preto/USP), Minas Gerais. Brasil.

$\triangle$ emerson93.ew@gmail.com. (D) https://orcid.org/0000-0002-6846-021X.

${ }^{6}$ Doutora em Saúde pública-Epidemiologia (UFMG), Professora (PPGCS/Unimontes), Minas Gerais. Brasil.

$\triangle$ martins.andreamebl@gmail.com. (i) https://orcid.org/0000-0002-1205-9910.

${ }^{7}$ Doutora em Ciência Animal - Epidemiologia (UFMG), Professora (Faculdade de Odontologia/UFMG), Minas Gerais. Brasil. $\square$ efigenia@uai.com.br.(D) https://orcid.org/0000-0002-0665-211X.

${ }^{8}$ Doutora em Odontologia em Saúde Coletiva (UFMG), Professora (PPGCS/Unimontes), Minas Gerais. Brasil.

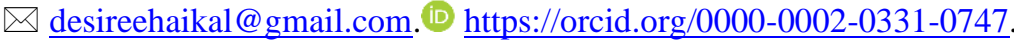

$\begin{array}{ccc}\text { Recebido em } & \text { Aceito em } & \text { Publicado em } \\ 09 / 12 / 2020 & 21 / 12 / 2020 & 31 / 12 / 2020\end{array}$


$(85,4 \%)$, apresentaram maiores proporções de casadas/união estável e divorciadas/viúvas, com filhos, de classe social mais baixa, perfil laboral precário, com melhores comportamentos relacionados à saúde, apesar de relatarem maior autopercepção negativa de sua aparência e pior qualidade de vida nos domínios físico e psicológico em relação aos homens. Já os homens apresentaram um melhor perfil laboral, maiores frequências de comportamentos não saudáveis e menor busca por assistência médica/odontológica. Observou-se consideráveis diferenças entre os gêneros, demonstrando que são as mulheres que refletem o maior peso da precarização profissional e os homens, os piores comportamentos de saúde. Desse modo, este estudo contribui para que as políticas públicas repensem as oportunidades sociais e questões de saúde relacionadas aos gêneros dentro da classe docente.

Palavras chaves: Perfil laboral; Comportamento; Saúde; Professores; Análise de gênero.

Abstract: The objective was to identify possible gender differences between teachers according to work profile, behaviors and health. This is a prevalence study using a probabilistic sample by conglomerates of primary school teachers from the public system of a municipality in northern Minas Gerais. Prevalence and bivariate analyzes were estimated, corrected by the sample design. Of the 745 teachers investigated, women represent a large majority (85.4\%), had higher proportions of married / stable union and divorced / widows, with children, of lower social class, precarious work profile, with better health-related behaviors, despite reporting greater negative self-perception of their appearance and worse quality of life in the physical and psychological domains in relation to men. Men, on the other hand, had a better work profile, higher frequencies of unhealthy behaviors and less search for medical / dental assistance. Considerable differences were observed between genders, demonstrating that it is women who reflect the greatest weight of professional insecurity and men, the worst health behaviors. Thus, this study contributes to public policies to rethink social opportunities and health issues related to genders within the teaching class.

Keywords: Work profile; Behavior; Health; Teachers; Gender analysis.

\section{INTRODUÇÃO}

O trabalho docente no Brasil, especialmente da educação básica de escolas públicas, é marcado por um processo de precarização e desvalorização da profissão ${ }^{1,2}$. As pressões sofridas no ambiente de trabalho do professor, como indisciplina de alunos, conflitos interpessoais, baixos salários e jornada excessiva de trabalho, representam fatores determinantes de baixas condições laborais entre esses profissionais $\mathrm{s}^{3,4,5}$.

A vivência diária com esses determinantes podem acarretar implicações no processo de aprendizagem dos alunos, além de interferir no cotidiano familiar, estilo de vida e prática de comportamentos não saudáveis ${ }^{3-6}$, tais como hábitos alimentares inadequados, vida sedentária e consumo de tabaco e álcool, já observados entre os professores de vários níveis do ensino brasileiro ${ }^{3,6,7}$. 
Além disso, o trabalho docente é fortemente marcado por relações sociais, como de gênero ${ }^{2}$. O termo "gênero" está ligado aos padrões culturais estabelecidos entre homens e mulheres, incorporando suas diferentes necessidades e papéis sociais ${ }^{8}$. Tais papéis interferem no processo de socialização individual e determinam valores, atitudes e condutas diferenciadas $^{8-10}$. Portanto, o termo ultrapassa a questão do "sexo", usualmente adotado apenas como um marcador de diferenças biológicas entre os indivíduos. Para além disso, as investigações sobre as diferenças de gênero consideram fatores complexos de vivências singulares entre homens e mulheres e almejam identificar o modo particular de ser e de agir de cada um ${ }^{10}$.

Estudos nacionais vêm demonstrando diferenças entre os gêneros no contexto educacional, que ultrapassam o grande predomínio do sexo feminino na profissão. Além da diferença salarial e de jornada laboral, têm sido observadas percepções distintas diante das responsabilidades e pressões sofridas no trabalho docente entre homens e mulheres ${ }^{2,11-14}$. No entanto, as diferenças das relações de gênero dentro do campo docente evidenciam uma desvalorização do docente do sexo feminino, visto que, apesar da carreira ser exercida majoritariamente por mulheres, há diferenças evidentes nas condições de trabalho ${ }^{15}$ e na qualidade de vida ${ }^{7}$.

A produção científica no campo das condições de trabalho e gênero ainda se mostra restrita ${ }^{2}$. Tal lacuna sinaliza a necessidade de estudos que possam desvelar, ainda mais, quem são esses sujeitos, quais são as condições de trabalho e os comportamentos de saúde que influenciam na qualidade de vida desses profissionais.

Partindo da premissa de que o ambiente escolar é um espaço de trabalho feminino, cabe avaliar se, em seu interior, há diferenças na valorização social do trabalho e nas relações sociais de gênero entre os homens e as mulheres.

Portanto, este estudo objetivou investigar o perfil laboral, comportamentos e saúde, segundo análise de diferenciais de gênero entre os professores da educação básica da rede pública de ensino de um município norte mineiro.

\section{MÉTODO}

Trata-se de estudo epidemiológico transversal, sendo seus dados extraídos a partir de dados do projeto matriz intitulado Projeto ProfSMoc: "Condições crônicas de saúde e 
fatores associados entre professores da rede pública estadual de Montes Claros-MG: estudo de base populacional", desenvolvido em Montes Claros, município polo localizado ao norte do Estado de Minas Gerais, de porte médio, que apresenta características de capital regional, com raio de influência que abrange todo o norte de Minas Gerais e parte do sul da Bahia, com contingente populacional estimado de 402.027 habitantes em $2017^{16}$.

\section{Amostragem}

Foi conduzida uma amostra probabilística por conglomerado em único estágio (escolas), sendo estimada a participação de 700 professores, considerando populações finitas (1856 docentes), prevalência de 50\% do evento de interesse, nível de confiança de 95\%, erro tolerável de $5 \%$ acrescido de $10 \%$ para compensar possíveis perdas e tendo sido adotado deff $=2,0$. Das 49 escolas estaduais urbanas presentes no município, foram sorteadas 35, considerando probabilidade proporcional ao tamanho. Todos os docentes vinculados a essas escolas com o exercício da função há pelo menos um ano, foram convidados a participar. Foram excluídos os professores em desvio de função ou de licença médica por qualquer natureza.

\section{Estratégia de coleta e análise dos dados}

A coleta de dados foi realizada no período de março a dezembro de 2016 por profissionais calibrados e treinados, mediante três etapas em cada escola sorteada. A primeira etapa foi o primeiro contato com os diretores e supervisores escolares; a segunda etapa caracterizou-se pela sensibilização dos professores acerca da pesquisa; A terceira e última etapa consistiu no recolhimento da assinatura do termo de consentimento livre esclarecido e dos questionários preenchidos, sendo realizadas a sua conferência e uma avaliação física.

O instrumento para coleta de dados foi um questionário autoaplicável proveniente do projeto matriz com questões elaboradas pelos próprios autores, descritas em todos os blocos temáticos, e questões relacionadas à atividade física e qualidade de vida a partir de instrumentos validados em estudos internacionais e nacionais, referenciadas nos blocos temáticos 4 e 5 . Neste estudo, foram consideradas as seguintes variáveis, apresentadas em blocos temáticos: 
1) Condições sociodemográficas e econômicas: idade; estado civil; cor de pele autodeclarada; possui filhos; e classe econômica. A variável idade foi coletada de forma numérica, em anos, e posteriormente categorizada pela mediana. A classe social foi avaliada através do Critério de Avaliação Econômica Brasil $2015^{17}$.

2) Perfil de formação acadêmica e ocupacional: nível de escolaridade; tempo de docência; rede de atuação; tipo de vínculo empregatício; carga horária semanal de trabalho; e trabalho remunerado além da docência.

3) Percepções relativas ao trabalho, subdivididas em: satisfação com o trabalho; desejo de mudar de profissão e incômodos autorreferidos relativos à violência por parte dos alunos; indisciplina dos alunos; falta de apoio das famílias dos alunos; superlotação de turma; infraestrutura e recursos materiais; insegurança; falta de capacitação; falta de colaboração da equipe; falta de apoio da escola; e salário.

4) Comportamento em saúde, subdividido em: hábitos de vida; e utilização dos serviços de saúde. Nos Hábitos de vida, considerou-se os autorrelatos de hábitos tabagista e etilista, consumo alimentar, horas diárias de TV, consumo de água e horas de sono. Quanto ao Consumo alimentar, foram investigados o número de dias da semana com ingestão de verduras/legumes, carnes com excesso de gorduras, fruta ou suco de fruta natural, refrigerante ou suco artificial, alimentos doces/guloseimas; a troca da comida do almoço ou jantar por sanduíches, salgados, pizza ou outros lanches; e o consumo de alimentos altamente $\operatorname{processados}^{18}$. Na sequência, foi conduzida análise de cluster pela técnica Two Step Cluster, na qual foram identificados dois padrões de consumo alimentar, sendo o primeiro considerado mais adequado por ter apresentado maior média diária de consumo de alimentos saudáveis e menor média de alimentos não saudáveis ${ }^{19}$. A atividade física foi aferida pelo Questionário Internacional de Atividade Física (IPAQ) na versão curta validada no Brasil ${ }^{20}$. As informações foram obtidas a partir da referência de uma semana habitual. Foram considerados ativos os indivíduos que fizeram 150 minutos ou mais de atividades físicas moderadas na semana, ou 75 minutos de atividades vigorosas ou combinação de ambas as intensidades em blocos de no mínimo 10 minutos $^{20}$. Os indivíduos que revelaram tempo de atividade física inferior aos mencionados foram agrupados na categoria sedentário/insuficientemente ativos. Quanto à utilização dos serviços de saúde, considerou-se o autorrelato da utilização do Sistema Único de Saúde (SUS); utilização do Instituto de Previdência dos Servidores de Minas Gerais 
(IPSEMG); possui plano de saúde ou convênio médico privado; tempo decorrido desde o último atendimento médico e odontológico.

5) Condições subjetivas de saúde: autopercepção de saúde geral (Atualmente, como você classificaria seu estado de saúde?); autopercepção da aparência (Quanto à aparência de seu corpo, você está...); autopercepção da saúde bucal (Atualmente, como você classificaria sua saúde bucal?); e qualidade de vida (QV). A QV foi avaliada pelo WHOQOL-Bref, versão resumida com validade em português do Instrumento de Avaliação da Qualidade de Vida da Organização Mundial de Saúde $(\mathrm{OMS})^{21}$. O WHOQOL-bref é constituído de 26 perguntas, sendo duas sobre a QV geral e as outras 24 compondo quatro domínios (físico, psicológico, social e meio ambiente). Quanto maior o escore, melhor a $\mathrm{QV}^{21}$. Como não tem sido recomendada a utilização de um ponto de corte padronizado para a QV, que é específico para cada população investigada, estimou-se as médias para cada domínio em separado e seus intervalos de $95 \%$ de confiança (IC-95\%). O valor obtido para o limite inferior do intervalo de confiança da média foi adotado como ponto de corte e os indivíduos cujos escores de cada domínio, bem como da QV geral se localizavam abaixo desse valor foram considerados com qualidade de vida insatisfatória ${ }^{22}$.

\section{Análise de dados}

Os dados foram digitados em duplicata, tabulados e analisados com auxílio do programa Statistical Package for Social Sciences (SPSS), versão 18.0. As análises foram conduzidas considerando apenas os professores que estavam atuando em sala de aula (regentes). As análises foram conduzidas segundo a variável sexo. Inicialmente, foram realizadas análises descritivas das variáveis, com a apresentação de frequências relativas (\%) e do Intervalo de Confiança de 95\% (IC-95\%). As frequências relativas foram estimadas respeitando-se a necessidade de correção pelo efeito de desenho, por serem provenientes de amostras por conglomerados. Para isso, a cada professor foi atribuído um peso, que correspondeu ao inverso de sua probabilidade de inclusão na amostra, considerando o efeito do conglomerado (escolas) e as taxas de não resposta. Na sequência, foram conduzidas análises bivariadas através do teste Qui-Quadrado de Pearson, a fim de identificar potenciais diferenças ( $\alpha$ de $5 \%$ ) discutidas à luz da desigualdade entre gêneros. 


\section{Questões éticas}

O estudo atendeu aos princípios éticos da Resolução do Conselho Nacional de Saúde (CNS) n466/2012, pois foi um ramo do Projeto ProfSMoc: "Condições crônicas de saúde e fatores associados entre professores da rede pública estadual de Montes Claros-MG: estudo de base populacional", o qual foi aprovado pelo Comitê de Ética em Pesquisa (CEP/ Unimontes, $\mathrm{n}^{\mathrm{O}}$ 1.293.458). Todos os participantes da pesquisa receberam e assinaram o Termo de Consentimento Livre e Esclarecido.

\section{RESULTADOS}

Participaram da coleta de dados 760 professores. No entanto, este estudo considerou os 745 que atenderam aos critérios de inclusão. A idade média observada foi de 40,5 anos $( \pm 9,6)$, e a renda per capita média de $\mathrm{R} \$ 1.496,50$ ( $\pm \mathrm{R} \$ 1.133,38)$. No geral, observou-se predomínio de professores do sexo feminino $(85,4 \%)$, casados/união estável $(62 \%)$, com filhos (69\%), com cor de pele autodeclarada parda (59\%), que se enquadraram na classe econômica B (62,2\%), que atuavam exclusivamente na rede pública $(91,5 \%)$, contratados/ designados $(58,4 \%)$ e com carga horária menor que 40 horas/semanais $(79,1 \%)$. Observaram-se diferenças significativas relativas ao gênero nas variáveis estado civil, possuir filhos, classe econômica, nível de escolaridade, redes de atuação na docência, vínculo com a escola e carga horária de trabalho (Tabela 1).

Tabela 1. Condições sociodemográficas e econômicas, perfil de formação acadêmica e ocupacional dos professores da rede básica do ensino público de Montes Claros/MG-2016.

\begin{tabular}{|c|c|c|c|c|c|c|c|}
\hline \multirow{2}{*}{ Variáveis } & \multicolumn{2}{|c|}{ Total } & \multicolumn{2}{|c|}{ Mulheres } & \multicolumn{2}{|c|}{ Homens } & \multirow[b]{2}{*}{$p$} \\
\hline & $\%$ & IC-95\% & $\%$ & IC- $-95 \%$ & $\%$ & IC-95\% & \\
\hline \multicolumn{8}{|c|}{ Sociodemográficas e econômicas } \\
\hline \multicolumn{8}{|c|}{ Idade (em anos) } \\
\hline$\leq 40$ & 50,3 & $45,7-54,8$ & 48,4 & $43,4-53,5$ & 61,2 & $52,6-69,1$ & \multirow{2}{*}{0,190} \\
\hline$>41$ & 49,7 & $45,2-54,3$ & 51,6 & $46,5-56,6$ & 38,8 & $30,9-47,4$ & \\
\hline \multicolumn{8}{|l|}{ Estado civil } \\
\hline Casado/União estável & 62,0 & $58,3-65,5$ & 64,3 & $60,3-68,0$ & 48,6 & $38,9-58,5$ & \multirow{3}{*}{$<0,001$} \\
\hline Solteiro & 27,1 & $23,4-31,2$ & 24,0 & $19,8-28,7$ & 45,7 & $37,3-54,3$ & \\
\hline Divorciado/Viúvo & 10,9 & $8,60-13,8$ & 11,8 & $9,10-15,1$ & 5,7 & $3,0-10,5$ & \\
\hline \multicolumn{8}{|c|}{ Cor da pele autodeclarada } \\
\hline Branca/ Amarela & 31,5 & $26,6-36,9$ & 30,9 & $25,7-36,7$ & 35,0 & $25,5-45,8$ & \multirow{3}{*}{0,508} \\
\hline Negra & 9,50 & $0,4-12,2$ & 9,4 & $7,0-12,4$ & 10,4 & $6,6-16,0$ & \\
\hline Parda & 59,0 & $54,7-63,1$ & 59,7 & $55,1-64,1$ & 54,6 & $45,6-63,4$ & \\
\hline \multicolumn{8}{|l|}{ Possui filhos } \\
\hline Não & 31,0 & $27,5-34,7$ & 27,7 & $23,9-31,8$ & 50,2 & $38,7-61,7$ & \multirow{3}{*}{$<0,001$} \\
\hline Sim & 69,0 & $65,3-72,5$ & 72,3 & $68,2-76,1$ & 49,8 & $38,3-61,3$ & \\
\hline Classe econômica & & & & & & & \\
\hline
\end{tabular}




\begin{tabular}{|c|c|c|c|c|c|c|c|}
\hline Classe A & 11,0 & $8,9-13,4$ & 10,1 & $8,6-13,6$ & 11,5 & $6,8-18,7$ & \\
\hline Classe B & 62,2 & $58,1-66,1$ & 60,2 & $55,6-64,6$ & 73,5 & $63,2-81,7$ & $\mathbf{0 , 0 1 3}$ \\
\hline Classes C e D/E & 26,9 & $22,8-31,4$ & 29,8 & $24,4-34,0$ & 15,0 & $8,6-24,9$ & \\
\hline \multicolumn{8}{|c|}{ Perfil de formação acadêmica e ocupacional } \\
\hline \multicolumn{8}{|c|}{ Nível de escolaridade } \\
\hline Mestrado/doutorado & 3,40 & $2,2-5,50$ & 2,6 & $1,5-4,60$ & 8,4 & $4,6-14,8$ & \multirow{3}{*}{0,001} \\
\hline Especialização & 51,8 & $48,1-55,5$ & 54,3 & $49,8-58,5$ & 37,8 & $29,3-47,2$ & \\
\hline Graduação & 44,6 & $40,8-48,6$ & 43,1 & $38,6-47,6$ & 53,8 & $45,6-61,8$ & \\
\hline \multicolumn{8}{|l|}{ Tempo de docência } \\
\hline $0-5$ & 27,3 & $23,5-31,5$ & 26,6 & $22,5-31,2$ & 31,3 & $24,4-39,2$ & \multirow{5}{*}{0,138} \\
\hline $5-10$ & 24,3 & $21,0-27,9$ & 23,7 & $20,2-27,5$ & 27,7 & $20,3-36,6$ & \\
\hline $10-15$ & 14,5 & $11,6-18,0$ & 14,3 & $11,0-18,3$ & 15,5 & $10,5-22,3$ & \\
\hline $15-20$ & 15,2 & $11,7-19,4$ & 15,4 & $11,5-20,3$ & 14,3 & $8,8-22,2$ & \\
\hline$>20$ & 18,7 & $15,6-22,3$ & 20,0 & $16,6-24,0$ & 11,2 & $6,7-18,0$ & \\
\hline \multicolumn{8}{|l|}{ Redes de atuação } \\
\hline Somente pública & 91,5 & $88,3-93,9$ & 92,9 & $89,7-95,1$ & 83,8 & $74,8-90,0$ & \multirow{2}{*}{0,002} \\
\hline Pública e privada & 8,5 & $6,1-11,7$ & 7,1 & $4,9-10,3$ & 16,2 & $10,0-25,2$ & \\
\hline \multicolumn{8}{|l|}{ Tipo de vínculo } \\
\hline Concursado/efetivo & 41,6 & $36,1-4,40$ & 39,3 & $33,6-45,3$ & 55,0 & $45,5-64,2$ & \multirow[t]{2}{*}{0,002} \\
\hline Contratado/designado & 58,4 & $52,6-68,9$ & 60,7 & $54,7-66,4$ & 45,0 & $35,8-54,5$ & \\
\hline \multicolumn{8}{|l|}{ Carga horária de trabalho } \\
\hline Menos de 40h & 79,1 & $73,6-83,6$ & 77,1 & $71,2-82,1$ & 90,6 & $84,7-94,4$ & \multirow{2}{*}{0,004} \\
\hline Mais de 40h & 20,9 & $16,4-26,4$ & 22,9 & $17,9-28,8$ & 9,40 & $5,6-15,3$ & \\
\hline \multicolumn{8}{|c|}{ Trabalho além da docência } \\
\hline Não & 78,3 & $75,0-81,2$ & 79,3 & $75,8-82,4$ & 72,1 & $63,5-79,4$ & \multirow{2}{*}{0,059} \\
\hline Sim & 21,7 & $18,8-25,0$ & 20,7 & $17,6-24,2$ & 27,9 & $20,6-36,5$ & \\
\hline
\end{tabular}

*Estimativas corrigidas pelo efeito do desenho amostral

Fonte: Próprios autores.

Quanto à satisfação dos professores com questões relacionadas ao trabalho, observou-se que, no geral, $17 \%$ dos professores estavam insatisfeitos e 15,2\% apresentavam desejo frequente de mudar de profissão. Os incômodos autorreferidos de maiores proporções pelos professores foram a violência por parte dos alunos $(62,6 \%)$, a indisciplina dos alunos (76,9\%), a falta de apoio da família do aluno $(77,6 \%)$ e o salário $(59,9 \%)$. Não foram encontradas diferenças significativas de gênero em relação à satisfação e ao desejo de mudar de profissão. Quanto aos incômodos autorreferidos relativos à profissão, observou-se maior proporção de mulheres muito incomodadas com a violência por parte dos alunos quando comparadas aos homens $(\mathrm{p}=0,019)$ (Tabela 2).

Tabela 2. Autopercepções relativas ao trabalho dos professores da rede básica do ensino público de Montes Claros/MG-2016.

\begin{tabular}{|c|c|c|c|c|c|c|c|}
\hline \multirow[t]{2}{*}{ Variáveis } & \multicolumn{2}{|r|}{ Total } & \multicolumn{2}{|c|}{ Mulheres } & \multicolumn{2}{|c|}{ Homens } & \multirow{2}{*}{$p$} \\
\hline & $\%$ & IC- $95 \%$ & $\%$ & IC-95\% & $\%$ & IC-95\% & \\
\hline \multicolumn{8}{|l|}{ Satisfação com o trabalho docente } \\
\hline \multicolumn{8}{|l|}{ Satisfação com o trabalho } \\
\hline Satisfeito & 41,5 & $36,2-47,0$ & 42,7 & $36,8-48,8$ & 34,8 & $26,1-40,6$ & \\
\hline Nem satisfeito nem insatisfeito & 41,6 & $37,1-46,3$ & 40,3 & $35,6-45,3$ & 49,1 & $41,1-57,1$ & 0,250 \\
\hline Insatisfeito & 16,9 & $13,5-20,8$ & 17,0 & $13,2-21,6$ & 16,1 & $11,0-23,0$ & \\
\hline \multicolumn{8}{|l|}{ Desejo em mudar de profissão } \\
\hline Nunca/Raramente & 35,8 & $31,5-40,3$ & 36,1 & $31,4-41,0$ & 34,2 & $27,1-42,1$ & \\
\hline Às vezes & 49,0 & $45,4-52,6$ & 49,8 & $46,2-53,5$ & 44,1 & $34,5-54,2$ & 0,225 \\
\hline Sempre/Frequentemente & 15,2 & $12,0-19,1$ & 14,1 & $10,8-18,3$ & 21,7 & $14,9-30,3$ & \\
\hline
\end{tabular}




\begin{tabular}{|c|c|c|c|c|c|c|c|}
\hline \multicolumn{8}{|l|}{ Incômodos autorreferidos } \\
\hline \multicolumn{8}{|l|}{ Violência dos alunos } \\
\hline Não existe/não incomoda & 19,6 & $15,6-24,3$ & 19,2 & $14,7-24,7$ & 21,7 & $14,8-30,7$ & \multirow{3}{*}{$\mathbf{0 , 0 1 9}$} \\
\hline Incomoda moderadamente & 18,2 & $15,5-21,3$ & 17,0 & $13,9-20,5$ & 25,6 & $18,4-34,5$ & \\
\hline Incomoda muito & 62,2 & $56,8-67,3$ & 63,8 & $58,0-69,2$ & 52,7 & $43,0-62,2$ & \\
\hline \multicolumn{8}{|l|}{ Indisciplina dos alunos } \\
\hline Não existe/não incomoda & 3,30 & $1,9-5,8$ & 3,6 & $2,0-6,4$ & 1,5 & $0,4-5,80$ & \multirow{3}{*}{0,143} \\
\hline Incomoda moderadamente & 19,8 & $16,4-23,6$ & 18,9 & $15,3-23,0$ & 25,2 & $18,5-33,3$ & \\
\hline Incomoda muito & 76,9 & $72,2-81,0$ & 77,5 & $72,2-82,1$ & 73,4 & $64,5-80,6$ & \\
\hline \multicolumn{8}{|l|}{ Falta apoio da família do aluno } \\
\hline Não existe/não incomoda & 5,0 & $3,60-6,90$ & 4,70 & $3,20-6,8$ & 6,5 & $2,90-14,1$ & \multirow{3}{*}{0,726} \\
\hline Incomoda moderadamente & 17,5 & $14,7-20,6$ & 17,3 & $14,5-20,5$ & 18,3 & $11,6-27,6$ & \\
\hline Incomoda muito & 77,6 & $73,5-81,2$ & 78,0 & $73,8-81,7$ & 75,2 & $63,6-84,1$ & \\
\hline \multicolumn{8}{|l|}{ Superlotação de turma } \\
\hline Não existe/não incomoda & 19,8 & $15,0-25,5$ & 20,5 & $15,6-26,6$ & 15,2 & $9,3-23,7$ & \multirow{3}{*}{0,228} \\
\hline Incomoda moderadamente & 28,0 & $24,8-31,4$ & 26,8 & $23,2-30,6$ & 35,2 & $26,1-45,5$ & \\
\hline Incomoda muito & 52,2 & $46,8-57,6$ & 52,7 & $47,0-58,3$ & 49,6 & $38,5-60,7$ & \\
\hline \multicolumn{8}{|c|}{ Infraestrutura e recursos materiais } \\
\hline Não existe/não incomoda & 14,2 & $11,5-17,5$ & 13,1 & $10,2-16,7$ & 20,8 & $13,3-30,9$ & \multirow{3}{*}{0,117} \\
\hline Incomoda moderadamente & 47,4 & $43,6-51,2$ & 47,8 & $43,2-52,4$ & 45,2 & $36,6-54,1$ & \\
\hline Incomoda muito & 38,4 & $34,2-42,8$ & 39,1 & $34,4-44,0$ & 34,1 & $27,5-41,3$ & \\
\hline \multicolumn{8}{|l|}{ Insegurança } \\
\hline Não existe/não incomoda & 27,6 & $23,6-32,1$ & 26,9 & $22,4-31,9$ & 31,8 & $22,9-42,2$ & \multirow{3}{*}{0,114} \\
\hline Incomoda moderadamente & 33,2 & $29,1-37,5$ & 32,3 & $27,6-37,4$ & 38,3 & $31,0-46,1$ & \\
\hline Incomoda muito & 39,2 & $34,9-43,7$ & 40,8 & $35,6-46,2$ & 30,0 & $20,5-41,5$ & \\
\hline \multicolumn{8}{|l|}{ Falta de capacitação } \\
\hline Não existe/não incomoda & 33,7 & $29,8-37,8$ & 33,5 & $29,1-38,2$ & 34,9 & $25,5-45,6$ & \multirow{3}{*}{0,702} \\
\hline Incomoda moderadamente & 39,6 & $35,6-43,9$ & 39,4 & $34,9-44,0$ & 41,4 & $32,9-50,4$ & \\
\hline Incomoda muito & 26,7 & $22,9-30,8$ & 27,2 & $23,2-31,6$ & 23,8 & $17,4-31,6$ & \\
\hline \multicolumn{8}{|l|}{ Falta colaboração da equipe } \\
\hline Não existe/não incomoda & 47,7 & $43,5-51,9$ & 47,0 & $41,9-52,2$ & 51,4 & $42,5-60,1$ & \multirow{3}{*}{0,070} \\
\hline Incomoda moderadamente & 33,7 & $30,6-37,0$ & 33,2 & $29,5-37,1$ & 36,8 & $30,4-43,8$ & \\
\hline Incomoda muito & 18,6 & $15,7-22,0$ & 19,8 & $16,5-23,5$ & 11,8 & $6,60-20,2$ & \\
\hline \multicolumn{8}{|l|}{ Falta apoio da direção da escola } \\
\hline Não existe/não incomoda & 58,1 & $53,5-62,7$ & 57,7 & $52,7-62,6$ & 60,7 & $48,8-71,4$ & \multirow{3}{*}{0,231} \\
\hline Incomoda moderadamente & 25,6 & $22,4-29,1$ & 25,3 & $21,9-29,1$ & 27,6 & $20,2-36,4$ & \\
\hline Incomoda muito & 16,2 & $13,5-19,4$ & 17,0 & $14,0-20,5$ & 11,8 & $6,70-19,9$ & \\
\hline \multicolumn{8}{|l|}{ Salário } \\
\hline Não existe/não incomoda & 10,0 & $7,90-12,7$ & 9,80 & $7,40-12,8$ & 11,7 & $6,30-20,4$ & \multirow{3}{*}{0,884} \\
\hline Incomoda moderadamente & 30,0 & $27,4-32,8$ & 29,9 & $27,1-32,9$ & 30,7 & $24,2-38,0$ & \\
\hline Incomoda muito & 59,9 & $55,8-64,0$ & 60,3 & $55,6-64,8$ & 57,6 & $50,0-65,0$ & \\
\hline
\end{tabular}

*Estimativas corrigidas pelo efeito do desenho amostral

Fonte: Próprios autores.

Em relação aos comportamentos em saúde, no geral, a classe docente investigada apresentou prevalência de hábito tabagista (2,0\%), consumo abusivo de álcool $(2,4 \%)$, consumo alimentar adequado (64\%) e de sedentário/insuficientemente ativos $(51,3 \%)$. Observaram-se diferenças significativas entre os gêneros. Homens apresentaram maiores proporções de comportamentos negativos quando comparados às mulheres, como hábito tabagista $(\mathrm{p}=0,003)$, hábito etilista $(\mathrm{p}=0,000)$, consumo alimentar inadequado $(\mathrm{p}=0,012)$, maior número de horas diárias de $\mathrm{TV}(\mathrm{p}=0,025)$ e menor busca por assistência médica $(\mathrm{p}=0,025)$ e odontológica $(\mathrm{p}=0,025)$ (Tabela 3$)$. 
Tabela 3. Comportamentos em saúde (Hábitos de vida e Utilização dos serviços) professores da rede básica do ensino público de Montes Claros/MG-2016.

\begin{tabular}{|c|c|c|c|c|c|c|c|}
\hline \multirow{2}{*}{ Variáveis } & \multicolumn{2}{|r|}{ Total } & \multicolumn{2}{|c|}{ Mulheres } & \multicolumn{2}{|c|}{ Homens } & \multirow[b]{2}{*}{$p$} \\
\hline & $\%$ & IC- $-95 \%$ & $\%$ & IC- $-95 \%$ & $\%$ & $\mathrm{IC}-95 \%$ & \\
\hline \multicolumn{8}{|l|}{ Comportamento em saúde } \\
\hline \multicolumn{8}{|l|}{ Hábitos de Vida } \\
\hline \multicolumn{8}{|l|}{ Hábito tabagista } \\
\hline Nunca fumou & 87,7 & $85,2-89,8$ & 89,3 & $86,8-91,3$ & 78,7 & $68,8-86,2$ & \multirow{3}{*}{$\mathbf{0 , 0 0 3}$} \\
\hline Ex-fumante & 10,3 & $8,6-12,3$ & 9,1 & $7,40-11,2$ & 16,9 & $10,7-25,6$ & \\
\hline Fumante & 2,00 & $1,2-3,4$ & 1,6 & $0,8-3,1$ & 4,40 & $1,8-10,2$ & \\
\hline \multicolumn{8}{|l|}{ Hábito etilista } \\
\hline Nunca /consumo esporádico & 80,9 & $77,6-83,9$ & 83,7 & $80,0-86,8$ & 64,8 & $55,1-73,4$ & \multirow{3}{*}{$<0,001$} \\
\hline Consumo frequente ( $1 \mathrm{x}$ semana) & 16,7 & $13,9-19,9$ & 15,0 & $12,1-18,4$ & 26,5 & $18,5-36,3$ & \\
\hline Consumo abusivo (3x semana) & 2,40 & $1,4-4,1$ & 1,30 & $0,5-3,4$ & 8,80 & $4,8-15,4$ & \\
\hline \multicolumn{8}{|l|}{ Consumo alimentar } \\
\hline Adequado & 64,0 & $59,7-68,1$ & 65,6 & $60,5-70,4$ & 54,5 & $46,0-62,7$ & \multirow{2}{*}{0,012} \\
\hline Inadequado & 36,0 & $31,9-40,3$ & 34,4 & $29,6-39,5$ & 45,5 & $37,3-54,0$ & \\
\hline \multicolumn{8}{|l|}{ Horas diárias de $T V$} \\
\hline Não assiste & 10,2 & $7,8-13,4$ & 10,6 & $7,8-14,2$ & 8,30 & $4,2-15,6$ & \multirow{3}{*}{$\mathbf{0 , 0 2 5}$} \\
\hline Assiste 2 horas ou menos & 70,1 & $66,8-73,2$ & 71,3 & $67,6-74,7$ & 63,1 & $55,1-70,5$ & \\
\hline Assiste 3 horas ou mais & 19,6 & $16,6-23,1$ & 18,1 & $15,0-21,7$ & 28,6 & $21,3-37,2$ & \\
\hline \multicolumn{8}{|l|}{ Consumo de água } \\
\hline Alto & 31,7 & $28,8-34,8$ & 29,9 & $27,0-32,9$ & 42,2 & $32,3-53,0$ & \multirow{3}{*}{$\mathbf{0 , 0 0 3}$} \\
\hline Moderado & 50,2 & $46,4-54,0$ & 50,4 & $45,9-54,9$ & 49,1 & $38,8-59,4$ & \\
\hline Baixo & 18,1 & $15,4-21,3$ & 19,8 & $16,6-23,3$ & 8,60 & $4,9-14,9$ & \\
\hline \multicolumn{8}{|l|}{ Atividade física } \\
\hline Sedentário/insuficientemente ativo & 51,3 & $46,8-55,6$ & 50,8 & $45,9-55,7$ & 53,8 & $42,2-64,9$ & \multirow{2}{*}{0,974} \\
\hline Ativo & 48,7 & $44,4-53,2$ & 49,2 & $44,3-54,1$ & 46,2 & $35,1-57,8$ & \\
\hline \multicolumn{8}{|l|}{ Horas de sono } \\
\hline Até 6 horas & 53,8 & $48,4-59,2$ & 53,4 & $47,6-59,2$ & 56,0 & $46,3-65,3$ & \multirow{2}{*}{0,690} \\
\hline 7 horas ou mais & 46,2 & $40,8-51,6$ & 46,6 & $40,8-52,4$ & 44,0 & $34,7-53,7$ & \\
\hline Utilização dos serviços de saúde & & & & & & & \\
\hline Utiliza SUS & & & & & & & \\
\hline Sim & 40,0 & $36,4-43,7$ & 39,1 & $35,1-43,2$ & 45,3 & $34,7-56,3$ & \\
\hline Não & 60,0 & $56,3-63,6$ & 60,9 & $56,8-64,9$ & 54,7 & $43,7-65,3$ & 0,331 \\
\hline Utiliza IPSEMG & & & & & & & \\
\hline $\operatorname{Sim}$ & 68,7 & $64,4-72,7$ & 69,9 & $65,4-74,2$ & 61,1 & $51,4-70,1$ & \\
\hline Não & 31,3 & $27,3-35,6$ & 30,1 & $25,8-34,6$ & 38,9 & $29,9-48,6$ & 0,027 \\
\hline Possui plano de saúde & & & & & & & \\
\hline $\operatorname{Sim}$ & 28,6 & $25,9-31,5$ & 30,6 & $27,6-33,7$ & 17,1 & $11,4-24,9$ & \\
\hline Não & 71,4 & $68,5-74,1$ & 69,4 & $66,3-72,4$ & 82,9 & $75,1-88,6$ & 0,003 \\
\hline Último atendimento médico & & & & & & & \\
\hline No último mês & 38,1 & $34,4-42,0$ & 39,3 & $35,4-43,4$ & 31,1 & $23,1-40,5$ & \\
\hline Entre um mês e um ano & 48,5 & $44,1-52,9$ & 48,1 & $43,4-52,9$ & 50,5 & $42,3-58,8$ & \\
\hline Entre um e dois anos & 9,9 & $7,9-12,4$ & 9,7 & $7,6-12,4$ & 10,9 & $7,2-16,3$ & $\mathbf{0 , 0 2 0}$ \\
\hline Mais de 2 anos & 3,5 & $2,4-5,1$ & 2,8 & $1,6-4,8$ & 7,4 & $4,3-12,5$ & \\
\hline Último atendimento odontológico & & & & & & & \\
\hline No último mês & 16,7 & $14,3-19,3$ & 18,2 & $15,5-21,4$ & 7,4 & $4,1-12,8$ & \\
\hline Entre um mês e um ano & 45,8 & $41,7-49,9$ & 45,8 & $41,1-50,6$ & 45,4 & $37,3-53,8$ & 0006 \\
\hline Entre um e dois anos & 24,5 & $21,3-27,9$ & 24,0 & $20,5-27,9$ & 27,2 & $20,0-35,7$ & 0,000 \\
\hline Mais de 2 anos & 13,1 & $10,4-16,4$ & 11,9 & $9,30-15,1$ & 20,0 & $12,6-30,3$ & \\
\hline
\end{tabular}

*estimativas corrigidas pelo efeito de desenho amostral.

Fonte: Próprios autores.

Em relação às condições subjetivas de saúde, no geral, 66,9\% da classe docente possui uma autopercepção positiva da saúde, 52,0\% com excelente/boa aparência corporal e 51,3\% declararam ter QV geral satisfatória. Os homens revelaram maior proporção de 
autopercepção positiva da aparência $(\mathrm{p}=0,000)$ e qualidade de vida satisfatória nos domínios físico $(\mathrm{p}=0,011)$ e psicológico $(\mathrm{p}=0,011)$ (Tabela 4).

Tabela 4. Condições subjetivas de saúde (Autopercepção de saúde e Qualidade de vida) dos professores da rede básica do ensino público de Montes Claros/MG-2016.

\begin{tabular}{|c|c|c|c|c|c|c|c|}
\hline \multirow[t]{2}{*}{ Variáveis } & \multicolumn{3}{|c|}{ Total } & \multicolumn{2}{|l|}{ Mulheres } & \multirow{2}{*}{$\begin{array}{c}\text { Homens } \\
\text { IC-95\% }\end{array}$} & \multirow{2}{*}{$p$} \\
\hline & $\%$ & IC-95\% & $\%$ & IC-95\% & $\%$ & & \\
\hline \multicolumn{8}{|c|}{ Condições subjetivas de saúde } \\
\hline \multicolumn{8}{|c|}{ Autopercepção da saúde geral } \\
\hline Excelente/boa & 66,9 & $62,9-70,7$ & 65,9 & $61,8-69,8$ & 73,1 & $63,2-81,1$ & \multirow{3}{*}{0,324} \\
\hline Regular & 27,9 & $24,5-31,5$ & 28,7 & $25,3-32,4$ & 22,8 & $15,4-32,3$ & \\
\hline Ruim/péssima & 5,20 & $3,8-7,0$ & 5,4 & $3,9-7,4$ & 4,10 & $1,30-12,3$ & \\
\hline \multicolumn{7}{|c|}{ Autopercepção da aparência } & \\
\hline Excelente/boa & 52,0 & $47,1-56,9$ & 50,8 & $45,3-56,3$ & 59,0 & $50,3-67,1$ & \multirow{3}{*}{$<0,001$} \\
\hline Regular & 11,1 & $9,4-13,1$ & 9,9 & $7,80-12,4$ & 18,4 & $12,6-26,1$ & \\
\hline Ruim/péssima & 36,9 & $32,4-41,5$ & 39,3 & $34,6-44,2$ & 22,6 & $15,4-32,0$ & \\
\hline \multicolumn{7}{|c|}{ Autopercepção da saúde bucal } & \multirow{4}{*}{0,443} \\
\hline Excelente/boa & 63,9 & $59,5-68,1$ & 63,8 & $58,9-68,3$ & 64,8 & $55,6-73,0$ & \\
\hline Regular & 29,4 & $26,1-33,0$ & 29,1 & $25,4-33,0$ & 31,4 & $22,9-41,5$ & \\
\hline Ruim/péssima & 6,7 & $5,0-9,0$ & 7,2 & $5,2-9,8$ & 3,80 & $1,8-8,0$ & \\
\hline \multicolumn{7}{|c|}{ QV geral (Qualidade de Vida) } & \multirow{3}{*}{0,090} \\
\hline Satisfatória & 51,3 & $46,5-56,1$ & 50,2 & $44,8-55,7$ & 57,5 & $48,8-65,6$ & \\
\hline Insatisfatória & 48,7 & $43,9-53,5$ & 49,8 & $44,3-55,2$ & 42,5 & $34,4-51,2$ & \\
\hline \multicolumn{7}{|l|}{ QV - Domínio físico } & \multirow{3}{*}{$\mathbf{0 , 0 1 1}$} \\
\hline Satisfatória & 55,1 & $50,4-59,6$ & 53,2 & $48,2-58,0$ & 66,2 & $56,8-74,4$ & \\
\hline Insatisfatória & 44,9 & $40,4-49,6$ & 46,8 & $42,0-51,8$ & 33,8 & $25,6-43,2$ & \\
\hline \multicolumn{7}{|l|}{ QV - Domínio psicológico } & \multirow{3}{*}{$\mathbf{0 , 0 1 1}$} \\
\hline Satisfatória & 58,6 & $54,3-62,7$ & 56,9 & $52,2-61,5$ & 68,1 & $59,7-75,5$ & \\
\hline Insatisfatória & 41,4 & $37,3-45,7$ & 43,1 & $38,5-47,8$ & 31,9 & $24,5-40,3$ & \\
\hline \multicolumn{7}{|l|}{$Q V$ - Domínio social } & \multirow{3}{*}{0,080} \\
\hline Satisfatória & 54,8 & $50,7-59,0$ & 53,6 & $49,0-58,2$ & 62,0 & $56,0-67,7$ & \\
\hline Insatisfatória & 45,2 & $41,0-49,3$ & 46,4 & $41,8-51,0$ & 38,0 & $32,3-44,0$ & \\
\hline \multicolumn{7}{|c|}{ QV - Domínio meio ambiente } & \multirow{3}{*}{0,072} \\
\hline Satisfatória & 50,8 & $46,6-55,0$ & 49,4 & $44,8-53,9$ & 59,2 & $50,0-67,7$ & \\
\hline Insatisfatória & 49,2 & $45,0-53,4$ & 50,6 & $46,1-55,2$ & 40,8 & $32,3-50,0$ & \\
\hline
\end{tabular}

\section{DISCUSSÃO}

Observou-se, no geral, uma classe docente de adulto jovem, com predomínio de autorrelato de cor parda, casados/união estável, com filhos e composta, em sua maioria, por mulheres. Houve predomínio de indivíduos da classe econômica B, com vínculo empregatício contratado/designado e com pós-graduação em nível de especialização. Esses dados se assemelham aos encontrados em outros estudos que investigaram a classe docente da rede pública do ensino básico da região sul do Brasil ${ }^{3,4}$. 
Neste estudo, observou-se diferenças significativas entre os gêneros no perfil laboral, comportamentos e saúde dos professores. Em relação às condições sociodemográficas e econômicas, as mulheres representam a maioria da população de professores e um inquérito nacional apontou a mesmo resultado na educação básica brasileira ${ }^{14}$. Esse fato parece contribuir com a vinculação da figura da professora aos aspectos de cuidado, afeto e maternidade ${ }^{15}$, principalmente nos anos iniciais da educação ${ }^{3,12}$. Observou-se, no grupo de mulheres, maior proporção de casadas/união estável e divorciadas/viúvas. Essas condições conjugais podem ser consideradas mais susceptíveis às sobrecargas de tarefas, pois frequentemente estão mais ligadas ao cuidado com a casa e com a família ${ }^{23}$. No caso das mulheres divorciadas e viúvas que criam filhos sozinhas, ocorre uma redução do acesso à melhores condições de vida em decorrência da necessidade de criação dos filhos ${ }^{24}$. Da mesma forma, a presença de filhos foi significativamente maior entre as mulheres neste estudo e esse resultado tem sido evidenciado frequentemente em outras pesquisas realizadas com a classe docente brasileira ${ }^{4,6,14}$.

A classe econômica que predominou neste estudo foi a classe $\mathrm{B}$, semelhante ao que foi encontrado em estudos anteriores sobre professores da rede municipal de Bagé/RS ${ }^{3} \mathrm{e}$ funcionários e professores de uma universidade comunitária do Sul do Brasil ${ }^{25}$. Todavia, neste estudo, observou-se um perfil econômico significativamente mais baixo entre as mulheres, condizente com estudo prévio entre professores da rede municipal de ensino de Vitória da Conquista/BA, que apontou que as mulheres possuíam menor qualificação, menores salários e menor status social ${ }^{2}$. Um estudo de diferenciais de gênero entre Brasil e França apontou que as mulheres tendem a ocupar posições inferiores e de menor prestígio, sendo que a disparidade salarial, a dificuldade para ascender na carreira e a segregação são problemas globais $^{26}$.

No que diz respeito ao perfil de formação acadêmica e ocupacional, observou-se, neste estudo, que a maioria dos professores possuía pós-graduação lato sensu (especialização), principalmente as mulheres. Quanto à pós-graduação stricto sensu (mestrado e doutorado), embora seja pequena a proporção de professores que a possui, ainda assim esse quesito foi comparativamente maior entre homens. Estudos com professores da rede pública do ensino básico na Bahia apontaram a hipótese de que os homens, até em funções predominantemente femininas, tendem a possuir nível de escolaridade mais elevado que as 
mulheres. Esse fato sugere que no trabalho docente, a maior qualificação formal ainda está sendo ocupada, preferencialmente, pelos homens ${ }^{2,11}$.

Neste estudo, revelou-se que, no geral, mais de $90 \%$ dos docentes trabalhavam somente na rede pública de ensino, sendo a maioria com carga horária docente inferior a $40 \mathrm{~h}$ semanais e contratada/designada (vínculo empregatício precário, sem estabilidade). Acreditase que a baixa remuneração e os contratos provisórios podem contribuir para a necessidade de busca de outras fontes de renda com acúmulo de jornada de trabalho, conforme observado em investigação prévia ${ }^{5}$. Os professores com contratos temporários não possuem garantias trabalhistas e previdenciárias, recebem menores salários,-através de uma relação de trabalho instável e informal ${ }^{4}$. É provável que essa situação reflita no processo de precarização da educação, pelo menos em parte, pois, independentemente do gênero, para se obter permanência do vínculo empregatício, é necessária a abertura de concursos públicos com a previsão da estabilidade no emprego, e a falta desse recurso se tornou uma realidade em muitas regiões brasileiras ${ }^{2,4}$.

As mulheres neste estudo apresentaram maior proporção de vínculo empregatício precário em relação aos homens, apesar de possuírem maior carga horária de trabalho docente. Um estudo conduzido entre docentes da rede municipal de ensino de Vitória da Conquista/BA revelou que eram os homens que possuíam maior proporção de vínculo precário em relação às mulheres, porém a maioria das mulheres trabalhava somente na rede pública e possuía maior carga horária em relação aos homens ${ }^{2}$. A superior carga horária de trabalho docente das mulheres em relação à dos homens também foi encontrada em estudos anteriores $^{1,2,3}$, estando o gênero feminino associado à maior ocorrência de desgaste profissional $^{2,27}$, maior surgimento de agravos à saúde $\mathrm{e}^{1,2,13,27}$, menor tempo para lazer e descanso, apresentando sono e alimentação prejudicados ${ }^{29}$ e com pior qualidade de vida geral $^{8}$. Além disso, os estudos destacaram que as mulheres se sentem responsabilizadas pelos afazeres domésticos e cuidados familiares, ${ }^{2,3}$ estando mais expostas às jornadas múltiplas e divididas entre a administração da casa, dos filhos, do trabalho e da formação acadêmica ${ }^{13,23}$. Ainda que a participação masculina no gerenciamento doméstico tenha aumentado, suas práticas e responsabilidades nas tarefas, muitas vezes, são complementares, desempenhando apenas um papel coadjuvante ${ }^{28}$.

Quanto à prevalência dos incômodos autorreferidos relativos à profissão, cerca de $2 / 3$ ou mais dos docentes relataram sentir-se "muito incomodados" com a violência e a 
indisciplina por parte dos alunos, o baixo salário e a falta de apoio da família do aluno. $\mathrm{Na}$ maioria dos quesitos investigados sobre os incômodos da profissão, não houve diferença entre os gêneros, exceto quanto à violência por parte dos alunos, que representou maior problema para as mulheres. Estudos nacionais que analisaram a violência contra a classe de professores revelaram que $76,5 \%$ dos professores no Paraná/PR ${ }^{29}$ e 97,3\% dos profissionais da educação em Natal/RN ${ }^{30}$ sofreram algum tipo de violência por parte dos alunos, sendo a maior prevalência destinada aos professores do gênero feminino ${ }^{29,30}$. O comportamento violento de alunos pode colaborar com a criação de um ambiente hostil, de medo e intimidação para professoras que atuam em escolas com esse perfil agressivo. Ademais, estudos que não fizeram diferenciação de gênero encontraram associação entre a violência estudantil e o estresse ocupacional com repercussão na saúde física e mental em educadores de São Luiz/MA ${ }^{31}$ e de Londrina/PR ${ }^{32}$. A categoria docente é uma das mais expostas a ambientes conflituosos e de alta exigência de trabalho. A violência verbal ou física e a indisciplina dos alunos são condições corriqueiras apontadas em estudos no contexto educacional ${ }^{4,6,32}$.

A classe docente, de maneira geral, revelou bons desempenhos quanto aos comportamentos em saúde, semelhante ao resultado encontrado em estudo com docentes do Rio Grande do $\mathrm{Sul}^{3}$. A prevalência geral observada de tabagismo (2\%) e etilismo $(2,4 \%)$ entre os docentes foi menor que as identificadas em adultos brasileiros, em que se observou aproximadamente $10 \%$ de tabagismo e $19 \%$ de etilismo ${ }^{18,33}$. Um estudo de base populacional com professores de vários estados brasileiros revelou baixa prevalência $(4,4 \%)$ de fumantes e apontou que o resultado positivo do estudo está relacionado, em parte, às campanhas educativas de combate ao tabagismo no Brasil, promoções de saúde escolar e à figura do professor, um exemplo vivo em sala de aula ${ }^{34}$.

Contudo, diferenças relacionadas aos gêneros foram constatadas. Homens apresentaram maiores frequências de comportamentos apontados como não saudáveis ${ }^{35}$ quando comparados às mulheres, com destaque aos hábitos tabagista, etilista, consumo alimentar inadequado e horas diárias de TV. Maiores prevalências do hábito tabagista ${ }^{34} \mathrm{e}$ etilista entre professores do gênero masculino já foram constatadas em outras investigações ${ }^{2,3}$. Talvez esses comportamentos nocivos à saúde do homem estejam atribuídos às diferenças nas exposições aos fatores e situações de risco ao longo da vida ${ }^{8,36}$, que contribuem para a maior vulnerabilidade dessa população devido ao baixo autocuidado e à pouca cultura de prevenção. 
Esse tipo de comportamento e a dedicação quase exclusiva ao trabalho podem colocar os homens em um quadro de negligência com sua saúde ${ }^{36}$.

A maior prevalência de consumo alimentar inadequado entre homens está em consonância com estudos nacionais com população adulta no Brasil, que apontaram que eles possuem maior prevalência no consumo de carnes com excesso de gordura ${ }^{37}$ e refrigerantes/ sucos artificiais ${ }^{18,33}$. Estudos apontaram que, em São Paulo ${ }^{25}$ e em Chapecó/SC ${ }^{38}$, houve elevado percentual de consumo alimentar irregular em profissionais da educação, porém sem diferenciação de gênero. A classe docente é caracterizada por uma jornada de múltiplos empregos e horários irregulares de trabalho ${ }^{12}$, rotina que, muito provavelmente, pode refletir na alimentação, propiciando maus hábitos alimentares com futuros problemas de saúde ${ }^{1-3}$.

Professores do sexo masculino relataram maior frequência em assistir a 3 horas ou mais de TV em relação às mulheres. Um inquérito de base populacional com população adulta das 26 capitais dos estados brasileiros e Distrito Federal apontou resultado divergente, com predomínio desse hábito entre as mulheres, sendo esse comportamento associado ao sedentarismo $^{39}$. Adicionalmente, outro estudo nacional com metodologia semelhante apontou esse hábito ao pior consumo alimentar de seus pesquisados ${ }^{40}$.

O baixo consumo de água foi predominante entre as mulheres deste estudo. Pesquisas com profissionais da educação de Passo Fundo/SP${ }^{25}$ e de Montes Claros/ $\mathrm{MG}^{41}$ reportaram baixo consumo de água em seus pesquisados, sendo que o último encontrou associação entre a pouca ingestão de água a disfonia aguda autorreferida. Possivelmente, esses resultados são devido à rotina agitada do contexto laboral do professor que, muitas vezes, desdobra-se para atender à várias instituições de ensino, tendo, inclusive, pouco tempo de deslocamento entre elas ${ }^{2,3}$.

Em relação à utilização dos serviços de saúde, diferenças significativas foram encontradas entre os gêneros, pois as mulheres utilizaram o IPSEMG e o plano de saúde/convênio privado com maior frequência que os homens. Da mesma forma, no que diz respeito ao último atendimento médico e odontológico, além da maior frequência, as mulheres utilizaram esses serviços em um espaço de tempo menor quando comparadas aos homens. Estudos nacionais desenvolvidos na Atenção Primária à Saúde encontraram baixa adesão dos homens, tanto nas consultas médicas ${ }^{42}$ quanto na assistência odontológica ${ }^{43}$. Outros estudos destacaram os principais motivos da baixa utilização dos serviços de saúde pelos homens, entre eles a influência da cultura da invulnerabilidade e força masculina, o desconhecimento 
sobre a importância do cuidado de si e a negligência com sua saúde ${ }^{44}$, bem como a falta de tempo, a incompatibilidade do horário de funcionamento dos serviços de saúde e o seu trabalho, o medo de descobrir doenças graves e o cuidado com sua saúde apenas em situações extremas $^{36}$. A utilização dos serviços de saúde apresenta um conjunto de determinantes, que vão desde a diferenciação de gênero na dimensão biológica à cultura de virilidade dos homens, chegando até ao excesso de autoconfiança ${ }^{8}$. Nesse sentido, os dados apresentados chamam atenção para a necessidade de estimular homens a se aproximarem dos serviços de saúde e adotarem hábitos de vida mais saudáveis.

Quanto à autopercepção da saúde e de sua aparência corporal, a maioria dos docentes investigados se autoavaliaram positivamente. Resultados análogos foram encontrados também em estudos nacionais conduzidos com professores da rede pública da educação básica ${ }^{2}$ e docentes universitários de uma faculdade particular ${ }^{12}$. No entanto, proporção importante $(40 \%)$ de mulheres autoavaliaram sua aparência corporal como ruim/péssima. Estudos sobre satisfação com a imagem corporal apontaram que 48,3\% na Alemanha $^{45}$ e $45,9 \%$ das mulheres investigadas no sul do Brasil ${ }^{46}$ estavam insatisfeitas com sua imagem corporal. Possivelmente, esses resultados seguem um relativo "padrão" entre mulheres, que parecem ser bastante autocríticas e excessivamente exigentes ao se perceberem, muitas vezes tendo como referência padrões de beleza dificilmente alcançados ${ }^{45,46}$. A crítica feminina em relação a sua imagem corporal reflete o contexto sociocultural atual muito influenciado pela mídia, associando a imagem de beleza feminina à magreza e juventude ${ }^{46}$. Essa questão merece atenção, visando aumentar a autoestima da mulher e diminuindo sua autocobrança.

$\mathrm{Na}$ qualidade de vida geral, quase metade dos participantes deste estudo se declararam insatisfeitos e resultado significante foi encontrado entre as mulheres, que tiveram percentuais insatisfatórios mais altos quando comparadas com os homens, principalmente nos domínios físico e psicológico. Estudo com professores do ensino fundamental e médio do Rio Grande do Sul revelou resultado semelhante ${ }^{6}$. Isso pode se dever ao fato de a classe docente ser marcada por condições precárias de trabalho ${ }^{2,4}$ e de as mulheres profissionais do ensino se sentirem mais responsabilizadas pela jornada doméstica e familiar ${ }^{2,23}$, com tempo reduzido para realizar atividades que lhes proporcionem lazer e prazer, o que acaba por afetar consideravelmente a sua qualidade de vida ${ }^{6,7}$. 
As desigualdades entre homens e mulheres são fomentadas pelas combinações culturais construídas socialmente, sendo adquiridas através da educação e aculturação ao longo da $\mathrm{vida}^{47}$. As diferenças mencionadas neste estudo corroboram os resultados encontrados da literatura e são decorrentes das relações de trabalho, do modo de vida e da construção sociocultural modulada pelas relações de gênero ao longo do tempo ${ }^{8,47}$.

Assim, este estudo enfatiza as diferenças determinadas pelo gênero, confirmando a relevância de analisar a variável sexo, tendo em vista que os estudos epidemiológicos são restritos nessa temática na classe docente ${ }^{15}$.

Este estudo apresenta limitações que precisam ser consideradas: a condução de análises bivariadas não permite a identificação de confundimento. Outra limitação importante foi não ter avaliado o nível de responsabilidade entre os gêneros das atividades domésticas e cuidados com os filhos, pois ao realizar a dupla jornada de trabalho pode ser um possível obstáculo ao avanço profissional, especialmente para as mulheres. Esta proposta é limitada aos profissionais de escolas da rede estadual do ensino básico de um município do norte de Minas Gerais. Assim, deve-se ter cautela ao extrapolar para outros subgrupos de trabalhadores ou de outras regiões brasileiras. Apesar das limitações, ressaltam-se pontos que aumentam a validade dos dados, com destaque para a calibração e concordância de toda a equipe de campo, amostra robusta, utilização de instrumentos validados e protocolos de avaliação, auditoria do banco de dados e análises corrigidas pelo desenho amostral. Além disso, as informações observadas permitiram identificar o lugar ocupado por homens e mulheres no contexto do trabalho docente. Assim, acredita-se que estudos dessa natureza sejam úteis e importantes para desnudar tais diferenças, a fim de minimizar as iniquidades, bem como de subsidiar planejamentos e ações de saúde voltadas para a classe docente com enfoque nas reais demandas manifestadas diferentemente por cada gênero.

Os achados encontrados também incitam futuras investigações de caráter longitudinal e qualitativo que possam integrar diferentes olhares à temática, a fim de elucidar ainda mais questões quanto às oportunidades profissionais, ao comportamento em saúde, às estratégias de enfrentamento no trabalho e à qualidade de vida dos professores sob a ótica da diferenciação de gênero. 


\section{CONCLUSÃO}

Conclui-se que importantes diferenças de gênero foram observadas no perfil laboral, de comportamentos e de saúde entre os professores, alcançando os objetivos propostos neste estudo. As mulheres deste estudo refletem o maior peso da precarização profissional, da autocobrança e da pior qualidade de vida. Já os homens, imperam comportamentos não saudáveis e menor frequência por assistência médica e odontológica.

Desse modo, sugere-se que as estratégias de enfrentamento dos problemas identificados precisam ser distintas e enfatiza que é impossível pensar a qualificação das professoras sem intervir em outras questões como o trabalho doméstico, o cuidado familiar e as inequidades profissionais a que são submetidas e, aos homens, a cultura do baixo cuidado de saúde. Assim, considerando que o ambiente escolar é marcado pela feminização da profissão, torna-se necessário estudos futuros que mergulhem no universo de questões ainda pouco exploradas, especialmente no campo das representações e construções simbólicas às questões de gênero dentro da atividade docente.

\section{REFERÊNCIAS}

1 GASPARINI, Sandra Maria; BARRETO, Sandhi Maria; ASSUNÇÃO, Ada Ávila. O professor, as condições de trabalho e os efeitos sobre sua saúde. Educação e Pesquisa, v. 31, n. 2, p.189-199, 2005.

2 ARAÚJO, Tânia Maria de et al. Diferenciais de gênero no trabalho docente e repercussões sobre a saúde. Ciênc. saúde coletiva, v. 11, n. 4, p. 1117-1129, 2006.

3 SANTOS, Márcio Neres dos; MARQUES, Alexandre Carriconde. Condições de saúde, estilo de vida e características de trabalho de professores de uma cidade do sul do Brasil. Ciênc. saúde coletiva, v. 18, n. 3, p. 837-846, 2013.

4 GUERREIRO, Natalia Paludeto et al. Perfil sociodemográfico, condições e cargas de trabalho de professores da rede estadual de ensino de um município da região sul do Brasil. Trabalho, educação e saúde, v. 14, supl. 1, p. 197-217, 2016.

5 COSTA, Gilvan Luiz Machado; OLIVEIRA, Dalila Andrade. Trabalho docente no ensino médio no Brasil. Perspectiva, v. 29, n. 2, p. 727-750, 2011. 
6 TABELEAO, Viviane Porto; TOMASI, Elaine; NEVES, Siduana Facin. Qualidade de vida e esgotamento profissional entre docentes da rede pública de Ensino Médio e Fundamental no Sul do Brasil. Cad. Saúde Pública, v. 27, n. 12, p. 2401-2408, 2011.

7 OLIVEIRA, Elizabete Regina Araújo de et al. Gênero e qualidade de vida percebida: estudo com professores da área de saúde. Ciênc. saúde coletiva, v.17, n.3, p. 741-747, 2012.

8 BARATA, Rita B. A posição social e seus reflexos sobre a saúde. In: Como e por que as desigualdades sociais fazem mal à saúde. Rio de Janeiro: Editora FIOCRUZ, 2009.

9 LUDERMIR, Ana Bernarda. Inserção produtiva, gênero e saúde mental. Cadernos de Saúde Pública, v.16, n. 3, p. 647-659, 2000.

10 BORRELL, Carme; ARTAZCOZ, Lucia. Desigualdades de gênero em saúde: desafios para o futuro. Rev. Esp. Saúde Pública, Madrid, v. 82, n. 3, p. 241-249, 2008.

11 DELCOR, Núria Serre et al. Condições de trabalho e saúde dos professores da rede particular de ensino de Vitória da Conquista, Bahia, Brasil. Cad. Saúde Pública, v. 20, n. 1, p. 187-196, 2004.

12 ROCHA, Kátia Bones; SARRIERA, Jorge Castellá. Saúde percebida em professores universitários: gênero, religião e condições de trabalho. Psicol. Esc. Educ. (Impr.),v. 10, n. 2, p. 187-196, 2006.

13 CARLOTTO, Mary Sandra et al. Transtornos Mentais Comuns (TMC) e fatores associados em trabalhadores: uma análise na perspectiva de gênero. Caderno Saúde Coletiva, v. 19, n. 2, p. 172-178, 2011.

14 ALCANTARA, Marcus Alessandro de et al. Determinantes de capacidade para o trabalho no cenário da Educação Básica do Brasil: Estudo Educatel, 2016. Cad. Saúde Pública, v. 35, supl. 1, e00179617, 2019.

15 NEVES, Mary Yale Rodrigues; BRITO, Jussara Cruz de; MUNIZ, Hélder Pordeus. A saúde das professoras, os contornos de gênero e o trabalho no Ensino Fundamental. Cad. Saúde Pública, v. 35, supl.1, e00189617, 2019.

16 IBGE-CIDADES. Instituto Brasileiro de Geografia e Estatística. Disponível em: <https://cidades.ibge.gov.br/v4/brasil/mg/montes-claros/panorama> Acesso em: Fevereiro/2020.

17 ABEP. Critério de Classificação Econômica Brasil. Associação Brasileira de Empresas de Pesquisa. 2015. 
18 BRASIL. Ministério da Saúde. Vigitel Brasil 2016: vigilância de fatores de risco e proteção para doenças crônicas por inquérito telefônico. Brasília, 2017.

19 VALli, Márcio. Análise de Cluster. Augusto Guzzo Revista Acadêmica, v. 4, p. 77-87, 2012.

20 MATSUDO, Sandra et al. Questionário internacional de atividade física (IPAQ): estudo de validade e reprodutibilidade no Brasil. Atividade Física \& Saúde, v. 6, n. 2, p. 5-18, 2001.

21 FLECK, Marcelo PA et al. Aplicação da versão em português do instrumento abreviado de avaliação da qualidade de vida "WHOQOL-bref". Revista de Saúde Pública, v. 34, n. 2, p.178-183, 2000.

22 UTAH DEPARTMENT OF HEALTH. Health status in Utah: the medical outcomes study SF-12 (2001 Utah health status survey report). Salt Lake City, 2004.

23 VERZA, Fabiana; SATTLER, Marli Kath; STREY, Marlene Neves. Mãe, mulher e chefe de família: perspectivas de gênero na terapia familiar. Pensando fam., v. 19, n. 1, p. 4660, 2015.

24 BEVILAQUA, Caroline; BUAES Caroline Stumpf. Sentidos de chefia familiar feminina em contextos de comunidades populares. Psicologia Argumento, v. 30, n. 68, p. 99-108, 2012.

25 ALVES, Ana Luisa Sant'Anna et al. Características do consumo alimentar de funcionários e professores de uma universidade comunitária. Arquivos de Ciências da Saúde, [S.1.], v. 24, n. 4, p. 42-46, 2017.

26 ABREU, Alice Rangel de Paiva; HIRATA, Helena; LOMBARDI, Maria Rosa. Gênero e trabalho no Brasil e na França: perspectivas interseccionais. Coautoria de Adriana Gracia Piscitelli. São Paulo, SP: Boitempo, 2016.

27 CNSDSS. Comissão Nacional sobre Determinantes Sociais da Saúde. As causas sociais das iniquidades em saúde no Brasil/Causes of social inequities in Brazil. Editora Fiocruz. 2008.

28 CARVALHO, Ana Maria Almeida et al. Mulheres e cuidado: bases psicobiológicas ou arbitrariedade cultural?. Paidéia, v. 18, n. 41, p. 431-444, 2008.

29 LEVANDOSKI, Gustavo; OGG, Fabiano; CARDOSO, Fernando Luiz. Violência contra professores de educação física no ensino público do estado do Paraná. Motriz: revista de educação física, v. 17, n. 3, p. 374-383, 2011. 
30 NETTO, Luciana et al. Experiências e especificidades da violência escolar na percepção de funcionários de uma escola pública. Revista de Enfermagem da UFSM, v. 2, n. 3, p. 591-600, 2012.

31 MESQUITA, Alex Andrade et al. Estresse e síndrome de burnout em professores: Prevalência e causas. Psicologia Argumento, v. 31, n. 75, p. 627-635, 2013.

32 KOGA, Gustavo Kenedy Camargo et al. Fatores associados a piores níveis na escala de Burnout em professores da educação básica. Cad. saúde Coletiva, v. 23, n. 3, p. 268$275,2015$.

33 BRASIL. Ministério da Saúde. Vigitel Brasil 2017: vigilância de fatores de risco e proteção para doenças crônicas por inquérito telefônico. Brasília, 2018.

34 BARBOSA, Rose Elizabeth Cabral; FONSECA, Giovanni Campos. Prevalência de tabagismo entre professores da Educação Básica no Brasil, 2016. Cad. Saúde Pública, v. 35, supl. 1, e00180217, 2019.

35 CDC. Centers for Disease Control and Prevention. Behavioral Risk Factor Surveillance System - BRFSS. About the BRFSS. Disponível em: https://www.cdc.gov/brfss/index.html. Acesso em: 08 jan. 2021.

36 ALVES, Railda Fernades et al. Gênero e saúde: o cuidar do homem em debate. Psicologia: teoria e prática, v. 13, n. 3, p. 152-166, 2011.

37 MALTA, Deborah Carvalho et al. Fatores de risco e proteção para doenças crônicas por inquérito telefônico nas capitais brasileiras, Vigitel 2014. Rev. bras. Epidemiologia, v. 18, Suppl. 2, p. 238-255, 2015.

38 GALLINA, Luciara Souza et al. Hábito alimentar do professor: importante elemento para a promoção da saúde no ambiente escolar. Revista Simbio-Logias, v. 6, n. 9, p. 105-116, 2013.

39 MIELKE, Grégore Iven et al. Prática de atividade física e hábito de assistir à televisão entre adultos no Brasil: Pesquisa Nacional de Saúde 2013. Epidemiol. Serv. Saúde, v. 24, n. 2, p. 277-286, 2015.

40 MAIA, Emanuella Gomes et al. Hábito de assistir à televisão e sua relação com a alimentação: resultados do período de 2006 a 2014 em capitais brasileiras. Cad. Saúde Pública, v. 32, n. 9, e00104515, 2016.

41 ROSSI-BARBOSA, Luiza Augusta Rosa et al. Self-Reported Acute and Chronic Voice Disorders in Teachers. Journal of Voice, v. 30, n. 6, p. 755, e25-755.e33, 2016. 
42 LEVORATO, Cleice Daiana et al. Fatores associados à procura por serviços de saúde numa perspectiva relacional de gênero. Ciênc. saúde coletiva, v. 19, n. 4, p. 1263 $1274,2014$.

43 SAINTRAIN, Maria Vieira de Lima et al. The relation between gender in the access to dental services and goods. Revista Brasileira Promoção à Saúde, v. 27, n. 3, p. 381$388,2014$.

44 SILVA, Patrícia Alves dos Santos et al. A saúde do homem na visão dos enfermeiros de uma unidade básica de saúde. Esc. Anna Nery, v.16, n. 3, p. 561-568, 2012.

45 LENGERKE, Thomas Von et al. Body weight dissatisfaction by socioeconomic status among obese, preobese and normal weight women and men: results of the cross-sectional KORA Augsburg S4 population survey. BMC Public Health, v.12, n. 342, p. 2-11, 2012.

46 POLTRONIERI, Taiara Scopel et al. Insatisfação com a imagem corporal e fatores associados em mulheres do sul do Brasil. Ciência \& Saúde, v. 9, n. 3, p. 128-134, 2016.

47 Krieger Nancy. Theories for social epidemiology in the 21st century: an ecosocial perspective. International Journal of Epidemiology, v. 30, n. 4, p. 668-677, 2001. 